\title{
Structured classification for ED presenting complaints - from free text field-based approach to ICPC-2 ED application
}

Tomi Malmström ${ }^{1 *+}$, Olli Huuskonen ${ }^{2+}$, Paulus Torkki $^{1+}$ and Raija Malmström ${ }^{3+}$

\begin{abstract}
Background: Although there is a major need to record and analyse presenting complaints in emergency departments (EDs), no international standard exists. The aim of the present study was to produce structured complaint classification suitable for ED use and to implement it in practice. The structured classification evolved from a study of free text fields and ICPC-2 classification.

Methods: Presenting complaints in a free text field of ED admissions during a one-year period $(n=40610)$ were analyzed and summarized to 70 presenting complaint groups. The results were compared to ICPC-2 based complaints collected in another ED. An expert panel reviewed the results and produced an ED application of ICPC-2 classification. This study implemented the new classification into an ED.

Results: The presenting complaints summarized from free text fields and those from ICPC-2 categories were remarkably similar. However, the ICPC-2 classification was too broad for ED; an adapted version was needed. The newly developed classification includes 89 presenting complaints and ED staff found it easy to use.
\end{abstract}

Conclusions: ICPC-2 classification can be adapted for ED use. The authors suggest a list of 89 presenting complaints for use in EDs adult patients.

\section{Background}

When a patient enters an emergency department (ED), important decisions are made at the very beginning of the visit concerning the necessity for, and the urgency of, medical examinations and care required by the patient. The presenting complaint, other anamnesis available, and a short status assessment determines the urgency of the treatment. Information from the referral notes or ambulance staff is also useful, if available. The presenting complaint is the patient's reason for the encounter, interpreted and recorded by the triage nurse. Although the importance of triage on patients' prognoses is recognized [1], there exists only sparse data on the presenting complaints of ED patients.

\footnotetext{
* Correspondence: tomi.malmstrom@aalto.fi

${ }^{\dagger}$ Equal contributors

'Institute of Healthcare Engineering and Management, Department of Industrial Engineering and Management, Aalto University, Otaniementie 17, 00076 Aalto, Finland

Full list of author information is available at the end of the article
}

Over the last 20 years, hospitals have developed different formal triage systems and these systems are in wide use [1]. No golden standard for triage exists, and careful attention should be paid on the studies, and the followups, of the competence and validity of the triage process in each ED. For example, studies on children [2], patients with unspecific complaints [3], and patients with sepsis [4] have shown the difficulties of triage.

To be able to study the impact of triage on the prognoses of different patient groups, structured information of the presenting complaints is needed. The process, from presenting complaint to diagnosis, is the core competence of emergency departments and failures at the beginning of the process - specifically, in triage - often lead to prolonged visits, endangered patient safety, and decreased patient satisfaction. Designing and controlling emergency departments with end diagnosis based information may result in inappropriate processes. A system that allows EDs to classify patients and define comparable case-mix groupings will help EDs describe their patient populations, workloads, staffing, and resource 
needs, and enable comparison across sites and regions [5]. In addition, it allows the development of automated decision support systems to specific patient groups, such as the automated evaluation of the Pneumonia Severity Index [6], or the reminder of stroke assessment form [7]. However, such a classification system should be relatively easy to adopt and implement, in order to be routinely utilized by ED staff.

No international standard for recording presenting complaints exists, and in many EDs, recording is not systematic. Even though the need for a systematic way to record presenting complaints was raised over a decade ago by Aronsky and colleagues [7], studies around the world report vast use of free-text fields and a general lack of a structured way to record presenting complaints (Australia [8,9]; US [10]; Finland [11]). The Canadian ED Information System (CEDIS) Working Group's Presenting Complaint List $[5,12]$ linked to the Canadian Triage and Acuity Scale (CTAS) has achieved good coverage in Canadian EDs. Other large international triage systems (ATS, MTS, ESI) do not provide structured classifications for PCs. Other than CEDIS, there are few other classifications presented in literature. Aronsky et al. [7] aimed at developing a generally applicable set of coded chief complaints for EDs and their study resulted in a list of 54 presenting complaints. Other classifications are presented in relation to single studies [3,8,13-17].

In contrast to fragmentation of presenting complaint classifications in EDs, there are established classifications for ambulatory care. Reason for Visit Classification (RVC) is used by the Centres for Disease Control and Prevention (CDC) and for the annually reported $\mathrm{Na}$ tional Hospital Ambulatory Care Survey (NHAMCS) in United States [18,19]. The International Classification for Primary Care (ICPC-2) is used for both diagnostic classification in family practice and primary care, and classification of ambulatory patients' Reasons for Encounter. WHO has accepted ICPC-2 within the WHO's Family of International Classifications (FIC), mainly as a method of encounter classification. ICPC-2 has been used in few studies within emergency services [20] and EDs [21,22]. However, neither RVC nor the ICPC-2 is ideal for use in EDs. Both systems are designed for primary care office hours and they each include several hundred complaints. (RVC includes 770 different complaints and the shortened version of the ICPC-2 has 687 codes for RFEs.)

The aim of the current study was to investigate whether the ICPC-2 could be adjusted to an ED presenting complaint classification, and thus, achieve an ED classification compatible with other areas of health care using the ICD-10 and the ICPC-2. The main reason for selecting the ICPC-2 was to employ an already internationally established classification as a foundation for the new classification. This study presents a structured classification for adult patients' ED presenting complaints based on ICPC-2 classification. The authors developed the ED presenting complaint classification by using free text analysis of 40,610 visits in one ED and by collecting presenting complaint data, using the ICPC-2, from 2,400 visits in another ED. Both EDs are responsible for primary care and special care urgent and emergent patients. An expert panel finalized the classification and the authors of this study implemented the new system in the Jorvi ED. This paper discusses the results.

\section{Terminology}

The presenting complaint in EDs refers to a professional interpretation of the symptoms or condition that made the patient seek emergency care. Presenting complaint is a term more established in Europe and Canada and its counterpart term in the U.S. is chief complaint (CC). Other terms sometimes used/mixed include Reason for Encounter (RFE), Reason for Visit, Presenting Problem, Problem on Admission, and Reason for Presenting. RFE refers to the pure reason for seeking medical advice, not including expert interpretation.

Figure 1 illustrates the use of presenting complaint terminology and related classification systems. A diagnostic classification is necessary in both elective and emergency care. Therefore, the ICD-10, with a broad diagnostic range, is used in both situations. The ICPC-2 was developed for diagnostic classification in family practice and primary care and it is based on the prevalence of health problems in primary care. The term patient's perspective represents the demand for care of that patient, and using the ICPC-2, it can be recorded as the reason for encounter both in elective and emergency care.

In emergency care, the triage nurse assesses the patient and makes decisions of urgency and requisite resources at the beginning of visits. In elective care, there is no need for the triage process or for the recording of the presenting complaint during the patient's visit. The presenting complaint should not be confused with RFE, although it is based partly on RFE.

\section{Methods}

\section{Study design}

The study included four phases and the overall design is illustrated in Figure 2. The objective of the first phase was to identify presenting complaints from free text fields of IT systems and to classify the presenting complaints into intuitive groups using one year data of an ED. Free text fields allowed identification of presenting complaints, as they were not biased by a classification system. 


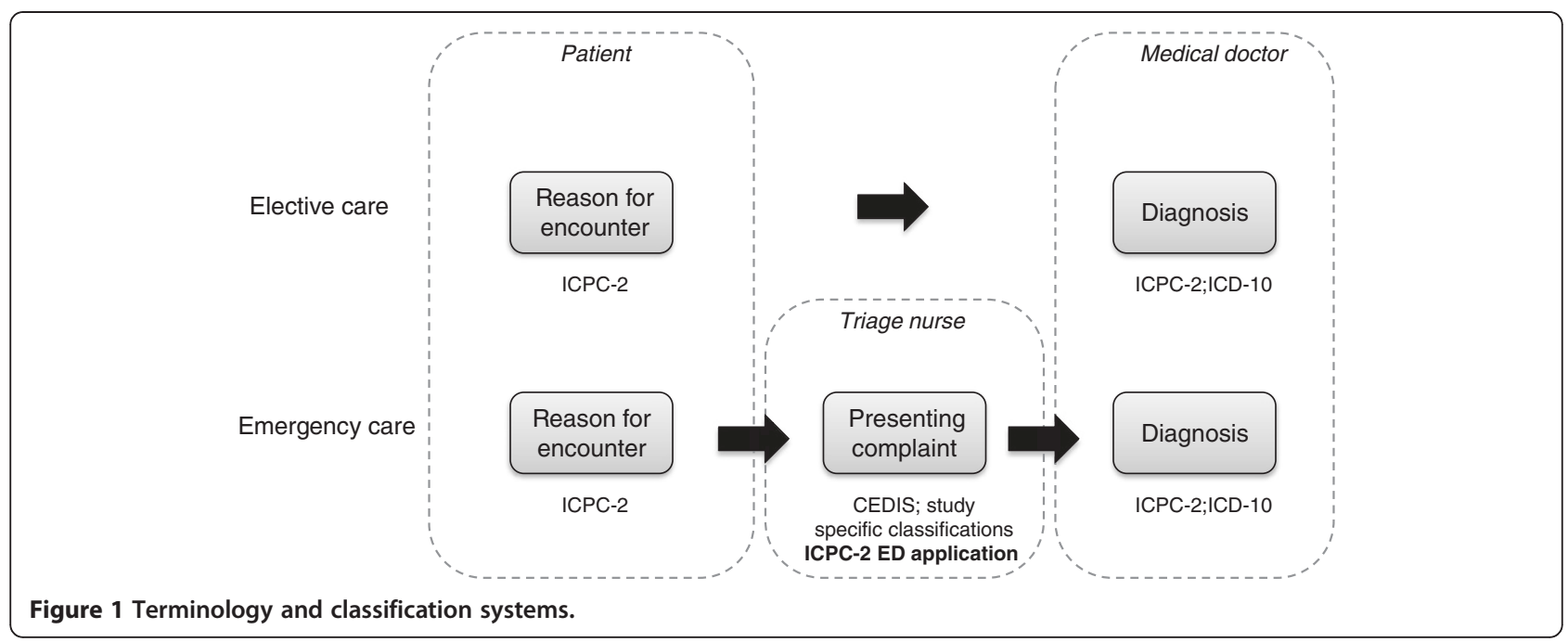

In the second phase, the objective was to implement the ICPC-2 classification in ED. As the ICPC-2 is an established classification system, mostly used in primary care diagnosis classification, the authors wanted to test its suitability for ED environments. The presenting complaints were collected with a two-week survey.

The third phase focused on creating an ICPC-2 based presenting complaint classification suitable for use in ED environments, and subsequently, in phase four to implement it in ED environments. The resulting classification system was based on the results of the first two phases and evaluated by an expert panel.

The study conducted the first phase in the Hyvinkää Hospital ED and the three others in the Jorvi Hospital ED. The following section presents both study sites and gives a more detailed description of each study phase.

\section{Study setting}

In Finland, 24/7 emergency services are mainly centralized to joint emergency units in charge of urgent and emergency services for special and primary health care. Two emergency units from Helsinki University Hospital District (HUCH) participated in this study, the Hyvinkää Hospital ED and the Jorvi Hospital ED.

The Hyvinkää Hospital ED serves a population of 185,000 inhabitants and the ED has 49,700 visits/year (2008). The Jorvi Hospital ED serves a population of 295,000 and has 62,500 visits/year (2010). During daytime hours, the health centres in the both regions also treat urgent primary care patients. Hyvinkää Hospital situates 50 kilometres, and Jorvi Hospital 15 kilometres, from the main University Hospital clinics in Helsinki. Patients with multiple injuries, candidates for thrombolytic therapy for stroke, and patients with an ST-elevation myocardial infarction were excluded from the study because according to $\mathrm{HUCH}$ policy, they were forwarded to the speciality emergency departments of the $\mathrm{HUCH}$ hospitals in Helsinki directly, by emergency services. In addition, childbirths were excluded from the study.

\section{Phase 1: identification of presenting complaints from free text field}

In the first phase, the objective was to identify presenting complaints of an ED using recordings from free text field. At Hyvinkää, the patients' presenting complaints are recorded in a free text field in the information system. If the patient comes without a referral, the presenting complaint is assessed by a nurse, based on the reason for the visit stated by the patient and possible information given by emergency services. If a patient has a referral, the presenting complaint is taken from the referral and entered by the department secretary. All presentations to the Hyvinkää ED during 2008 were included in the study.

Typically, if a patient is transferred from a general practitioner to a physician in specialized medical care

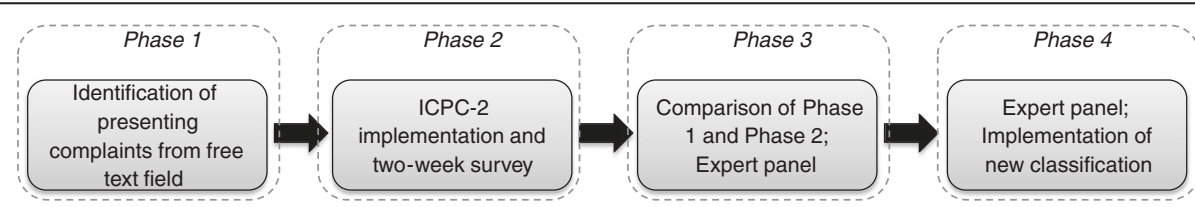

Figure 2 Overall study design. 
because of a consultation, a presenting complaint is entered in the system. However, this study used the original reason for encounter entered when the patient first came to the emergency clinic.

The data were reworked by selecting the presenting complaint for each patient in the free text of the field. If several reasons for encounter were entered for a single patient, the one requiring the most urgent care was selected. The prioritised reasons were shortness of breath, chest pain, and abdominal pain, respectively. Functions in the spreadsheet application MS Excel 2007 were used to select the reasons for encounter. The data rework was done by RM and TM.

In most of the cases, obvious presenting complaints could be picked up from the free text, and therefore, grouping of the synonyms and trimming of the different writing formats comprised the majority of the work. Unclear presenting complaints were discussed case by case, and the reason selected was based on a clinician's assessment (RM). If the presenting complaint could not be determined from the field, or if the field contained just individual symptoms, the encounter was excluded from the list.

\section{Phase 2: ICPC-2 implementation and two-week survey}

In the second phase, the objective was to implement the ICPC-2 classification to ED and evaluate its suitability to ED use. This phase was carried out in the Jorvi Hospital ED and data were collected from a survey. In Jorvi, ICPC-2 classification was already familiar, because visits to primary care had been recorded by triage nurses using ICPC-2 since 2007. However, the use of classification had not been systematic, and the concept of presenting complaint and diagnosis had been mixed. Researchers administered the survey over a continuous 14-day period, from 8:00 am Monday, November 15 to 8:00 am Monday, November 292010.

The presenting complaints were recorded on a paperformat questionnaire at the point of triage using ICPC-2 codes. To help staff record different ICPC-2 codes for presenting complaints, a two-page summary of codes was available at the triage facility. The two-page document is available in several languages on the WONCA website (http://www.globalfamilydoctor.com/wicc/) and it includes 687 different codes. Staff was allowed to record several presenting complaints for a single patient. However, the data analysis used only the most urgent presenting complaint.

\section{Phase 3: development of new ICPC-2 based ED application}

In the third phase, the objective was to develop a new ICPC-2 based ED application for recording the presenting complaints. The results of the first two phases were analysed and compared to see how case mix and presenting complaints differed between the Jorvi ED and the Hyvinkää ED. Jorvi's ICPC-2 based presenting complaints were coded to respond to the categories of the Hyvinkää ED's study. The presenting complaints from both sites were used for the frequency comparison.

Using the data of these two EDs and experiences of ICPC-2 use in Jorvi the expert panel discussions defined the new classification for use in the ED. The panel consisted of three senior medical doctors and one nurse. TM made notes and participated to the discussion. The expert panel first reviewed the results of phases 1-3 and used the ICPC- 2 based classification system. The panel also made use of other presenting complaint lists $[5,12,16,17]$ where it was suitable and the panel members modified the ICPC-2 presenting complaint list based on the discussions. The most important criteria in selecting presenting complaints were prevalence, urgency and possible streaming to different patient pathways.

The short, two-page version of the ICPC-2 classification already included 687 RFEs; therefore, in most cases, the panel grouped overly detailed complaints into one more general group.

To preserve compatibility with the original ICPC-2, the original codes remained. However, complex codes, such as F01-F04, F13-F16, and F28-F29 would be difficult for staff to use, and most of the IT-systems could not make such an exception. Therefore, in implementation, they were changed for the Jorvi ED by using only the numerical first code of the group (in the previous example of F01, F02, F03, and F04, the code would be F01).

Presenting complaints of patients with multiple injuries, candidates for thrombolytic therapy for stroke, and patients with ST-elevation myocardial infarctions who were originally excluded from the study were taken into consideration in the new classification.

\section{Phase 4: implementation of ICPC-2 ED application}

In the fourth phase, the objective was to implement the new ICPC-2 based ED application to Jorvi ED and to test the suitability of the classification. Before implementation of the new classification system, briefing sessions were organized for the staff. Most of the staff was familiar with the original ICPC-2 list, which made the adoption easier. Feedback from the staff was collected in weekly routine staff meetings by the head nurse in open discussions, and confidentially by a feedback box, and with one-to-one interviews conducted by RM. The staff was encouraged to give feedback from every case were they had difficulties in identifying easily an appropriate code. After two weeks use of the ICPC-2 ED application, a series of interviews captured staff experiences with the system. In total, 12 persons were interviewed one-to one 
by RM. Each nurse was asked the following three questions:

- Question 1. Are you satisfied with the new classification?

- Question 2. Have you had patients with presenting complaint which you have had difficulties to classify? If yes, please specify.

- Question 3. Have you some suggestions for further development of classification or other comments?

\section{Results}

Results from phase 1: identification of presenting complaints from free text field

There were 49,700 patient presentations in 2008 in the Hyvinkää ED and 40,610 of them were adults (16 or over). Complete data were available in 35,334 (87,0\%) patient encounters and used in the presenting complaint analysis.

The data categorization resulted in a list of 65 presenting complaints. There were 5,051 visits where the presenting complaint could not be determined. Out of these, 1,516 came for control visit due to recent visit in the ED and had only limited information about presenting complaint. In few cases the presenting complaint was due to rare disease or was related to very specific symptoms and in such a case it was not meaningful to form they own groups. The rest of the visits had either non-comprehensible or not presenting complaint related value in the free-text field. In addition, there were 225 empty presenting complaint fields. Therefore, 5,276 encounters were excluded $-13.0 \%$ of all visits. There were 9,300 patients ( $18.8 \%$ of all visits) with a referral.

Table 1 presents the presenting complaints used in the Hyvinkää ED and their frequencies.

\section{Results from phase 2: ICPC-2 implementation and two- week survey}

During the two-week survey, there were 2332 presentations at the Jorvi ED, of which 1309 (56,1\%) were in primary care and $1023(43,9 \%)$ in secondary care. A complete data set was gathered from 1837 presentations $(78,8 \%)$. Incomplete data was due to missing values in patient IDs, ages and presenting complaint information. Out of 1837 visits, 1284 were adults (16 or over) and were included to the study. Table 2 presents the divisions of presenting complaints from the Jorvi ED among general categories of the ICPC- 2 .

At the Jorvi ED, 254 (237 for adults) out of 687 different ICPC codes were used to record presenting complaints during the two-week survey period. Figure 3 shows the coverage achieved by the number of presenting complaint codes used. The amount is dependable on volume, and therefore, different curves are given for a day (second Monday, $\mathrm{n}=72$ ), a week (second week, $n=604)$, and the total period $(n=1284)$. The two-week period represented presenting complaints quite well; a longer period, with larger volume, would not have radically increased the number of presenting complaints. Coverage of 70\%-90\% was achieved from 50-120 different ICPC-2 based presenting complaints.

The experiences of using ICPC-2 showed in practise, that it was difficult to find a representative code for presenting complaints from ICPC-2 code list in ED. The list was far too long and included large number of codes not needed in ED. Regardless the fact that some codes require information not available at the point of triage, these codes (such as pneumonia and pulmonary embolism) were used anyway. Although the results from the phase 2 demonstrated the unsuitability of ICPC-2 classification in the raw for ED environments, it showed that ICPC-2 can be used as a basis of the ED classification. This conclusion was supported by the results of phase 3 showing that ICPC-2 classification include almost all the presenting complaints revealed in Hyvinkää data in phase 1.

\section{Results from phase 3: development of new ICPC-2 based ED application}

Table 3 illustrates differences in the most common presenting complaints between the Jorvi ED and the Hyvinkää ED. Presenting complaints of both EDs are relatively close to each other. The biggest differences in Hyvinkää, compared to Jorvi, are in presenting complaints related to trauma mechanisms such as electric shock or carbon monoxide poisoning, for which the ICPC-2 classification does not elaborate. Some of Hyvinkää's presenting complaints are also more diagnoses-based, such as pneumonia and urinary tract infection. In addition, in the Hyvinkää ED, the triage nurse sometimes recorded that patients came simply for a doctor's certificate of sick leave. In Finland, most organizations allow sick leaves of 2-3 days without a certificate, but EDs are often used for sick leaves exceeding that because of the convenience of access.

The classification of presenting complaints produced from the Hyvinkää ED's free text fields classifies all musculoskeletal symptoms and complaints under one group. Such a generic group becomes too large, covering over one fifth of all the presenting complaints.

\section{Results from phase 4: implementation of ICPC-2 ED application}

The staff experiences about implemented ICPC-2 ED application are described in the following.

- Question 1. Are you satisfied with the new classification?

All interviewed nurses answered yes. 
Table 1 Presenting complaints and frequencies in Hyvinkää ED

\begin{tabular}{|c|c|c|c|c|c|}
\hline Presenting complaint & Amount & Percentage & Presenting complaint & Amount & Percentage \\
\hline Musculoskeletal symptoms/complaints & 8596 & $21,17 \%$ & Blood pressure related problem & 198 & $0,49 \%$ \\
\hline Abdominal pain & 3084 & $7,59 \%$ & Intravenous antibiotic infusion & 183 & $0,45 \%$ \\
\hline Upper respiratory infection/throat symptom & 2862 & $7,05 \%$ & Bronchus/lung related symptom & 182 & $0,45 \%$ \\
\hline Shortness of breath & 1567 & $3,86 \%$ & Allergic reaction & 163 & $0,40 \%$ \\
\hline Chest pain & 1503 & $3,70 \%$ & Rash & 149 & $0,37 \%$ \\
\hline Diarrhoea/vomiting & 1492 & $3,67 \%$ & Blood and blood forming organs & 127 & $0,31 \%$ \\
\hline Cardiac arrhythmia & 1206 & $2,97 \%$ & Nose bleed & 123 & $0,30 \%$ \\
\hline Back symptom & 1161 & $2,86 \%$ & Icterus or ascites & 118 & $0,29 \%$ \\
\hline Fever & 1079 & $2,66 \%$ & Nausea & 102 & $0,25 \%$ \\
\hline Headache & 906 & $2,23 \%$ & Complication of medical treatment & 98 & $0,24 \%$ \\
\hline General weakness & 873 & $2,15 \%$ & For sick leave & 93 & $0,23 \%$ \\
\hline Infection of urinary tract & 823 & $2,03 \%$ & Haemorrhoid & 77 & $0,19 \%$ \\
\hline Eye symptom & 672 & $1,65 \%$ & Burn injury & 72 & $0,18 \%$ \\
\hline Symptoms of venous embolism & 652 & $1,61 \%$ & Neurological symptoms & 65 & $0,16 \%$ \\
\hline Psychological problems & 609 & $1,50 \%$ & Pregnancy related problem & 65 & $0,16 \%$ \\
\hline Vertigo/dizziness & 589 & $1,45 \%$ & Constipation & 64 & $0,16 \%$ \\
\hline Cough & 570 & $1,40 \%$ & Female genital symptoms & 61 & $0,15 \%$ \\
\hline Ear symptoms & 524 & $1,29 \%$ & Sleep disturbance & 55 & $0,14 \%$ \\
\hline Pneumonia symptoms & 452 & $1,11 \%$ & Symptoms of chronic bowel disease & 49 & $0,12 \%$ \\
\hline Cerebrovascular disorder symptoms & 393 & $0,97 \%$ & High value of C-reactive protein & 46 & $0,11 \%$ \\
\hline Convulsion & 389 & $0,96 \%$ & Catheter related & 34 & $0,08 \%$ \\
\hline Urological symptoms & 380 & $0,94 \%$ & Dental & 31 & $0,08 \%$ \\
\hline Blood test for drunk driving suspect & 344 & $0,85 \%$ & Cramp & 30 & $0,07 \%$ \\
\hline Intoxication & 299 & $0,74 \%$ & Trigeminal neuralgia & 23 & $0,06 \%$ \\
\hline Cancer related symptom & 298 & $0,73 \%$ & Tremor/shivering & 22 & $0,05 \%$ \\
\hline Drug or alcohol abuse & 295 & $0,73 \%$ & Tick & 20 & $0,05 \%$ \\
\hline Symptoms of acute sinusitis & 270 & $0,66 \%$ & Electric shock & 17 & $0,04 \%$ \\
\hline Lump or abscess & 256 & $0,63 \%$ & Hypothermia & 16 & $0,04 \%$ \\
\hline Skin infection & 247 & $0,61 \%$ & Dehydration & 12 & $0,03 \%$ \\
\hline Heart related problem, other & 212 & $0,52 \%$ & Carbon monoxide poisoning & 7 & $0,02 \%$ \\
\hline Fainting/syncope & 212 & $0,52 \%$ & Exposure to gas & 7 & $0,02 \%$ \\
\hline Endocrine/metabolic symptoms & 210 & $0,52 \%$ & Other & 5276 & $12,99 \%$ \\
\hline
\end{tabular}

- Question 2. Have you had patients with presenting complaint which you have had difficulties to classify? If yes, please specify.

All nurses answered that the suitable code was found for all presenting complaints. Two nurses reported that if a patient has many complaints, it is difficult to decide the main presenting complaint. One nurse asked, if it could be possible to get a code for pneumonia as a presenting complaint. Information of using the most urgent presenting complaint was further addressed to triage nurses as well as the problem of too early decision of possible diagnosis.

- Question 3. Have you some suggestions for further development of classification or other comments?
Four nurses asked for more feedback of the results of analysis of presenting complaints. They found it important to get reports to motivate the recording.

In the weekly staff meetings people felt that the new classification was easy to use and staff members found all the presenting complaints with no difficulties except the cases with many complaints. The staff was satisfied with the new system. The original ICPC-2 list had been in use in the Jorvi ED, and therefore, the staff compared the new list to the original ICPC-2 list. They had complained that the original ICPC-2 list was too long and that the right presenting complaints were difficult to locate. As the new list was based on the ICPC-2 list, the staff felt immediately familiar with the codes. They also 
Table 2 Use of general categories of the ICPC-2 in Jorvi ED

\begin{tabular}{llll}
\hline $\begin{array}{l}\text { General } \\
\text { category }\end{array}$ & Name & Amount & Share \\
\hline- & Process codes & 6 & $0,47 \%$ \\
\hline $\mathrm{A}$ & General and unspecified & 172 & $13,40 \%$ \\
\hline $\mathrm{B}$ & $\begin{array}{l}\text { Blood, Blood Forming Organs } \\
\text { and immune Mechanism }\end{array}$ & 6 & $0,47 \%$ \\
\hline $\mathrm{D}$ & Digestive & 172 & $13,40 \%$ \\
\hline $\mathrm{F}$ & Eye & 12 & $0,93 \%$ \\
\hline $\mathrm{H}$ & Ear & 13 & $1,01 \%$ \\
\hline $\mathrm{K}$ & Cardiovascular & 149 & $11,60 \%$ \\
\hline $\mathrm{L}$ & Musculoskeletal & 275 & $21,42 \%$ \\
\hline $\mathrm{N}$ & Neurological & 87 & $6,78 \%$ \\
\hline $\mathrm{P}$ & Psychological & 75 & $5,84 \%$ \\
\hline $\mathrm{R}$ & Respiratory & 141 & $10,98 \%$ \\
\hline $\mathrm{S}$ & Skin & 93 & $7,24 \%$ \\
\hline $\mathrm{T}$ & Endocrine/Metabolic and & 10 & $0,78 \%$ \\
\hline $\mathrm{N}$ & Nutritional & & \\
\hline $\mathrm{X}$ & Urological & 50 & $3,89 \%$ \\
\hline $\mathrm{Y}$ & Pregnancy, Childbearing, & 13 & $1,01 \%$ \\
\hline $\mathrm{F}$ & Family Planning & & \\
\hline $\mathrm{W}$ & Female genital & 2 & $0,16 \%$ \\
\hline & Male genital & 4 & $0,31 \%$ \\
\hline & Social problems & & \\
\hline & & & \\
\hline
\end{tabular}

felt that the length and groupings were improvements to the previous list.

The feedback from the staff generated still a few adjustments regarding hyperglycaemia and delirium, which are included in final list in Table 4. The final list includes 89 presenting complaints. The list is now in routine use in Jorvi ED and the recording percent is high (over $95 \%$ ).

\section{Discussion}

This study created a structured complaint classification suitable for ED use and implemented it to practice. The classification is based on two stand-alone studies; one focused on identifying presenting complaints from data of 40,610 ED presentations and the other on a two-week survey while using the ICPC-2 as a classification for recording presenting complaints. The study produced a classification based on the ICPC-2, including 89 presenting complaints. The results from implementation were positive and encouraging and the classification is currently in routine use in the Jorvi ED.

Two major factors motivated the selection of the ICPC-2 as the foundation of the new classification. Firstly, the ICPC-2 is symptom-oriented. In the triage process, patients have no predefined diagnoses per se, and the triage nurse's should base his/her interpretation of urgency and requisite resources on symptoms. Secondly, the ICPC-2 is compatible with the ICD-10. The ICPC-2 is a part of WHO's Family of International Classifications (FIC) and all of the complaints can be traced back to related ICD-10 codes. However, the ICPC-2 has too much detail for effective use in EDs.

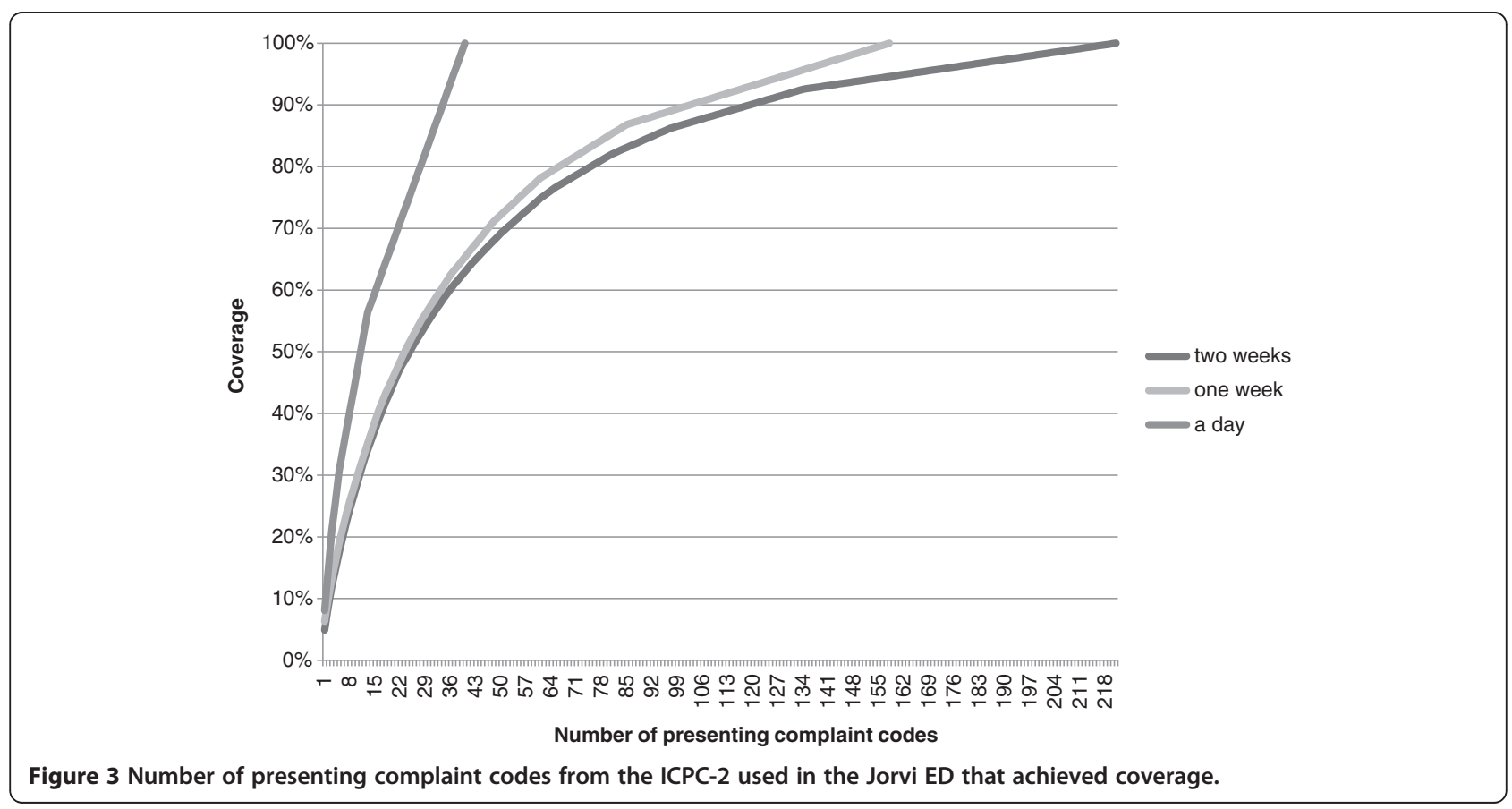


Table 3 Top 20 presenting complaints in Hyvinkää ED and in Jorvi ED

\begin{tabular}{|c|c|c|c|}
\hline $\begin{array}{l}\text { Top } 20 \text { presenting complaints in Hyvinkää ED } \\
\text { (Year 2008) }\end{array}$ & Share & $\begin{array}{l}\text { Top } 20 \text { presenting complaints in Jorvi ED } \\
\text { (two weeks, Nov-2010) }\end{array}$ & Share \\
\hline Musculoskeletal symptoms/complaints & $21,17 \%$ & Musculoskeletal symptoms/complaints & $20,43 \%$ \\
\hline Abdominal pain & $7,59 \%$ & Abdominal pain & $8,38 \%$ \\
\hline Upper respiratory infection/throat symptom & $7,05 \%$ & Psychological problems & $4,11 \%$ \\
\hline Shortness of breath & $3,86 \%$ & Shortness of breath & $3,89 \%$ \\
\hline Chest pain & $3,70 \%$ & Chest pain & $3,89 \%$ \\
\hline Diarrhoea/vomiting & $3,67 \%$ & General weakness & $3,82 \%$ \\
\hline Cardiac arrhythmia & $2,97 \%$ & Fever & $3,75 \%$ \\
\hline Back symptom & $2,86 \%$ & Skin infection & $3,31 \%$ \\
\hline Fever & $2,66 \%$ & Back symptom & $3,23 \%$ \\
\hline Headache & $2,23 \%$ & Upper respiratory infection/throat symptom & $3,09 \%$ \\
\hline General weakness & $2,15 \%$ & Cardiac arrhythmia & $2,87 \%$ \\
\hline Infection of urinary tract & $2,03 \%$ & Headache & $2,20 \%$ \\
\hline Eye symptom & $1,65 \%$ & Diarrhoea/vomiting & $2,06 \%$ \\
\hline Symptoms of venous embolism & $1,61 \%$ & Infection of urinary tract & $1,76 \%$ \\
\hline Psychological problems & $1,50 \%$ & Convulsion & $1,25 \%$ \\
\hline Vertigo/dizziness & $1,45 \%$ & Vertigo/dizziness & $0,96 \%$ \\
\hline Cough & $1,40 \%$ & Ear symptoms & $0,96 \%$ \\
\hline Ear symptoms & $1,29 \%$ & Eye symptom & $0,96 \%$ \\
\hline Pneumonia symptoms & $1,11 \%$ & Female genital symptoms & $0,88 \%$ \\
\hline Cerebrovascular disorder symptoms & $0,97 \%$ & Fainting/syncope & $0,73 \%$ \\
\hline
\end{tabular}

A short list of presenting complaints is simple and reliable, but if the list is too short, it does not present enough information. When the list is long, the specificity is higher but the system is complex and data analysis becomes difficult. With a long list, shortened modifications emerge and their use jeopardizes data comparison. ED-specific presenting complaint lists range between 33 [16] and 165 [12] in the number of different codes. The current study indicates that a list of 89 presenting complaints is well suited for ED use. In triage, time for recording codes is scarce; the system has to be easy to use. Obtaining presenting complaint information from a free text field is very time-consuming and not suitable for routine use.

Unlike the ICPC-2 based classification of this study, Canadian CEDIS classification is ED-specific. Although many presenting complaints are similar in both lists, there are remarkable differences. The main difference in this study's shorter list is that the presenting complaints of many organs, such as ear, eye, or gynaecological symptoms, are not divided into as many subclasses as those in the CEDIS; patients in these subclasses have the same pathways and use the same resources in EDs. However, the study's list included more subclasses to code traumas and symptoms of the extremities; symptoms of the ankle, knee, and hip have their own codes. Rare but urgent cases, such as periorbital oedema, were omitted because the inclusion of one such item would soon increase the list with other equally urgent, but rare, symptoms. One must keep in mind that almost all presenting complaints can include emergent cases.

In some cases the CEDIS list had more interpretation of the symptoms than did the list in the current study. The study list, for example, listed hyperventilation with dyspnoe to prevent premature conclusions of possible diagnoses. Hyperventilation can be a harmless symptom, but it can also be a symptom of ketoasidosis or pulmonary embolism. This same principle led to the decision to keep chest pain as one presenting complaint without trying to divide it into more or less specific cardiac features. This is important because a cardiac event is difficult to diagnose [23].

The ICPC-2 was quite easily modified and suites the classification of ED presenting complaints well. Many codes for the ED list were combined from two or three codes of the ICPC-2. However, some symptoms had to be modified from the ICPC-2. Hyperglycaemia is under abnormal investigation results in the ICPC-2, while hypoglycaemia has its own code and allergic reaction, which the new list had to include despite its features of diagnosis and the fact that it is coded as a diagnosis in the ICPC-2.

Classification of presenting complaints does not remove the need for using free text communication in EDs, it is highly important and codes or rules should not restrict its use. In the Jorvi Hospital ED, the presenting 
Table 4 ICPC-2 ED application

\begin{tabular}{|c|c|c|c|}
\hline $\begin{array}{l}\text { ICPC-2 codes } \\
\text { included }\end{array}$ & Name & $\begin{array}{l}\text { ICPC-2 codes } \\
\text { included }\end{array}$ & Name \\
\hline General & & Neurological & \\
\hline $\mathrm{A} 01$ & Pain general/multiple sites & N01, N03 & Headache; Pain face \\
\hline A03 & Fever & N05-06 & Sensation disturbance \\
\hline A04-A05 & Weakness/tiredness general, feeling ill & N07 & Convulsion/seizure \\
\hline A06 & Fainting/syncope & N17 & Vertigo/dizziness \\
\hline $\mathrm{A} 07$ & Coma & N18 & Paralysis/weakness \\
\hline A80-A81 & Trauma/injury NOS, multiple trauma & N19 & Speech disorder \\
\hline A87 & Complication of surgical or other treatment & N29 & Delirium \\
\hline A88 & Adverse effect physical factor & \multicolumn{2}{|c|}{ Psychological/Toxic effects } \\
\hline A91 & $\begin{array}{l}\text { Abnormal result investigation NOS; } \\
\text { Hyperglycaemia }\end{array}$ & P01 & Feeling anxious/nervous/tense \\
\hline A92 & Allergy/allergic reaction NOS & P02 & Acute stress reaction \\
\hline A96 & Death & P03 & Feeling depressed \\
\hline Digestive & & P15-P16 & Alcohol abuse \\
\hline D01-D02, D06 & Abdominal pain/cramps general & P18 & Medication abuse \\
\hline D03 & Heartburn & P19 & Drug abuse \\
\hline D04-D05 & Rectal/anal pain or itching & P20 & Memory disturbance \\
\hline D09-D10 & Nausea, vomiting & P29 & Psychological symptom/complaint other \\
\hline D11, D18 & Diarrhoea & P77 & Suicide/suicide attempt/suicidality \\
\hline $\mathrm{D} 12$ & Constipation & A84 & Poisoning by medical agent \\
\hline D13 & Jaundice & A86 & Toxic effect non-medicinal substance \\
\hline $\mathrm{D} 14$ & Haematemesis/vomiting blood & \multicolumn{2}{|l|}{ Respiratory } \\
\hline D15-D16 & Melaena & R01-R04 & $\begin{array}{l}\text { Shortness of breath, dyspnoea, } \\
\text { pain respiratory system, wheezing, } \\
\text { breathing problem }\end{array}$ \\
\hline D19 & Teeth/gum symptom/complaint & R05 & Cough \\
\hline $\mathrm{D} 20$ & Mouth/tongue/lip symptom/complaint & R06 & Nose bleed \\
\hline D25, D29 & Abdominal distension & R07-R21, R28-R29, R74 & Nose/sinus/throat/voice symptom/complaint \\
\hline Eye & & R24 & Haemoptysis \\
\hline F01-F03, F13-F16, F29 & Eye symptoms/complaints & \multicolumn{2}{|l|}{ Skin } \\
\hline F04-05 & Visual disturbance & S01, S02,S04-S08, S99 & $\begin{array}{l}\text { Pain/tenderness of skin; Pruritus; } \\
\text { Lump/swelling; Rash }\end{array}$ \\
\hline Ear & & S10-S11 & Boil/carbuncle, skin-infection posttraumatic \\
\hline $\mathrm{H} 01-\mathrm{H} 05, \mathrm{H} 13, \mathrm{H} 29$ & Ear symptoms/complaints & $\mathrm{S} 12$ & Insect bite/sting \\
\hline Cardiovascular & & $\mathrm{S} 13$ & Animal/human bite \\
\hline $\mathrm{A} 11, \mathrm{~K} 01-\mathrm{K} 03$ & Chest pain, pressure/tightness of heart & S14 & Burn/scald \\
\hline K04-K05 & $\begin{array}{l}\text { Palpitations/ awareness of heart; Bradykardia; } \\
\text { Irregular heartbeat }\end{array}$ & S15 & Foreign body in skin \\
\hline $\mathrm{K} 07$ & Swollen ankle/oedema & $\mathrm{S} 18$ & Laceration/cut \\
\hline K29 & $\begin{array}{l}\text { Cardiovascular symptom/complaint other, } \\
\text { high blood pressure }\end{array}$ & \multicolumn{2}{|l|}{ Endocrine/Metabolic } \\
\hline Musculoskeletal & & $\mathrm{T} 11$ & Dehydration \\
\hline L01 & Neck symptom/complaint & T27 & Hypoglycaemia \\
\hline L02-L03 & Back symptom/complaint & \multicolumn{2}{|l|}{ Urological } \\
\hline L04 & Chest symptom/complaint & U01, U02, U07, U14 & Dysuria/painful urination \\
\hline L05 & Flank/axilla symptom/complaint & U06 & Haematuria \\
\hline L07 & Jaw symptom/complaint & U08 & Urinary retention \\
\hline L08 & Shoulder symptom/complaint & \multicolumn{2}{|l|}{ Pregnancy } \\
\hline
\end{tabular}


Table 4 ICPC-2 ED application (Continued)

\begin{tabular}{llll}
\hline L09 & Arm symptom/complaint & W03 & Antepartum bleeding \\
\hline L10 & Elbow symptom/complaint & Female genital & \\
\hline L11 & Wrist symptom/complaint & $\begin{array}{l}\text { X01, X03, X08, X09, X12, } \\
\text { X14-X17 }\end{array}$ & Female genital symptoms \\
\hline L12 & Hand/finger symptom/complaint & X18 & Breast symptoms \\
\hline L13 & Hip symptom/complaint & Male genital & \\
\hline L15 & Leg/thigh symptom/complaint & Y01-Y06 & Male genital symptoms \\
\hline L16 & Knee symptom/complaint & Social problems & \\
\hline L17 & Ankle symptom/complaint & Z25 & Assault/harmful event/ problem \\
\hline L18-L19 & Foot/toe symptom/complaint & Z29 & Social problem \\
\hline & Muscle pain; Muscle symptom/complaint & Process codes & \\
\hline & NOS & -50 & Medication/prescription/renewal/injection \\
\hline
\end{tabular}

complaint code is not used for communication at all; it is registered only for purposes of data analysis and quality studies and free text is used for communication.

Decisions made in triage regarding urgency and tracks have significant effects on the duration and the quality of the care process. The presenting complaint is one of the most important variables affecting these decisions. The variety of patients entering EDs is wide, and to evaluate triage performance and care quality, divisions between different patient groups are necessary. Often studies concerning EDs make divisions and give treatment recommendations based on diagnoses or treatments but such information is not available at the point of triage. Presenting complaints provide more relevant divisions for studying quality, process, and outcomes of care; for such research, structured information regarding presenting complaints is essential.

In addition, routine research in the classification of presenting complaints in ERs enables several practical data usage possibilities. Presenting complaint information, for example, aids demand and capacity planning, streaming, and patient flow control, quality control, and benchmarking of performance.

\section{Limitations}

Although the studied EDs represent medium-sized joint EDs, which are typical to Finnish healthcare system, there may be need for customization and for more detailed presenting complaints classification in highly specialized units. Moreover, the new classification was tested only in one ED and to ensure reliability and validity with different case-mixes further studies may be needed.

It should be noted that the evaluation method of new classification is not strictly following any qualitative method and is not reported according to any qualitative standard.
All classifications need continuous improvement. Therefore, our study classification is not suitable for broad use without centralized national or international actor for development. The study focused only in adult patients and the classification is not directly generalizable to presenting complaints of children.

\section{Conclusions}

The ICPC-2 classification can be easily modified for use in EDs by decreasing the number of codes. The use of structured classification for recording presenting complaints in EDs helps to compare and improve EDs, both nationally and internationally. Recording presenting complaints provides information from ED case-mixes and helps in the planning and control of patient flows.

It is essential that future research develops a similar, ICPC-2 based presenting complaint list for pediatric emergency care. Further validation studies should also be conducted to the classification in different environments using standard evaluation protocols such as COREQ [24].

The authors recommend the use of the ICPC-2 based list for recording ED presenting complaints; the new list with 89 presenting complaints was more convenient and easy to use. The classification is easily transferable to different EDs, although such a classification needs to be developed and improved continually. To ensure data comparability, all such improvement modifications should be centralized, either by national actor or by an international organization willing to take the classification forward, such as the WONCA International Classification Committee.

Competing interests

The authors declare that they have no competing interests. TM and PT are employed by Nordic Healthcare Group, NHG. NHG is a commercial company that focuses on healthcare and welfare industries and designs models to enhance productivity, cost-effectiveness and process quality. The business is 
based on research and has employees in Stockholm, Sweden and Helsinki, Finland.

\section{Authors' contributions}

TM designed the study, conducted the data analysis for Hyvinkää and Jorvi data, and drafted the manuscript. OH helped in the data collection (Jorvi and expert panel) and revised the manuscript. PT helped in the data analysis and revised the manuscript. RM co-designed the study, organized the data collection (Hyvinkää, Jorvi, and expert panel), performed the interviews, and helped to draft the manuscript. All authors read and approved the final manuscript.

\section{Acknowledgements}

The authors express their gratitude to RN Kristina Nyholm and MD Salla Kumpulainen for participating on the expert panel and MD Juha Valli and M. Sc Sirpa Salinto for Hyvinkää Hospital data.

\section{Author details}

${ }^{1}$ Institute of Healthcare Engineering and Management, Department of Industrial Engineering and Management, Aalto University, Otaniementie 17, 00076 Aalto, Finland. ${ }^{2}$ Jorvi Hospital, Division of Emergency Care, Meilahti Hospital, Helsinki University Hospital District, Helsinki, Finland. ${ }^{3}$ Department of Medicine, Division of Emergency Care, Meilahti Hospital, Helsinki University Hospital District, Helsinki, Finland.

Received: 11 May 2012 Accepted: 22 November 2012

Published: 24 November 2012

\section{References}

1. FitzGerald G, Jelinek GA, Scott D, Gerdtz MF: Emergency department triage revisited. Emerg Med J 2010, 27:86.

2. Nijman RG, Zwinkels RL, Van Veen M, Steyerberg EW, Van Der Lei J, Moll HA, Oostenbrink R: Can Urgency Classification of the Manchester Triage System Predict Serious Bacterial Infections in Febrile Children? Arch Dis Child 2011, 96:715-722.

3. Nemec M, Koller MT, Nickel CH, Maile S, Winterhalder C, Karrer C, Laifer G, Bingisser R: Patients Presenting to the Emergency Department With Nonspecific Complaints: The Basel Non-specific Complaints (BANC) Study. Acad Emerg Med 2010, 17:284-292.

4. Cronshaw HL, Daniels R, Bleetman A, Joynes E, Sheils M: Impact of the Surviving Sepsis Campaign on the Recognition and Management of Severe Sepsis in the Emergency Department: Are We Failing? Emerg Med J 2011, 28:670-675.

5. Grafstein E, Unger B, Bullard M, Innes G: Canadian Emergency Department Information System (CEDIS) Presenting Complaint List (Version 1.0). Can J Emerg Med 2003, 5:27.

6. Fine MJ, Auble TE, Yealy DM, Hanusa BH, Weissfeld LA, Singer DE, Coley CM, Marrie TJ, Kapoor WN: A prediction rule to identify low-risk patients with community-acquired pneumonia. New Engl J Med 1997, 336:243-250.

7. Aronsky D, Kendall D, Merkley K, James BC, Haug PJ: A comprehensive set of coded chief complaints for the emergency department. Acad Emerg Med 2001, 8:980-989.

8. Wagholikar AS, Lawley MJ, Hansen DP, Chu K: Identifying Symptom Groups from Emergency Department Presenting Complaint Free Text using SNOMED CT. AMIA Annu Symp Proc 2011, 2011:1446-1453.

9. Hansen DP, Kemp ML, Mills SR, Mercer MA, Frosdick PA, Lawley MJ: Developing a national emergency department data reference set based on SNOMED CT. Med J Aust 2011, 194:S8-S10.

10. Matheny ME, FitzHenry F, Speroff T, Green JK, Griffith ML, Vasilevskis EE, Fielstein EM, Elkin PL, Brown SH: Detection of infectious symptoms from VA emergency department and primary care clinical documentation. Int J Med Inform 2012, 81:143-156.

11. Malmström T, Torkki P, Valli J, Malmström R: Patient flow analysis of Hyvinkää Hospital joint emergency unit. Finnish Med J 2012, 5:345-351. in Finnish.

12. Grafstein E, Bullard MJ, Warren D, Unger B: Revision of the Canadian Emergency Department Information System (CEDIS) Presenting Complaint List version 1.1. Can J Emerg Med 2008, 10:151-173.

13. Gupta M, Tabas JA, Kohn MA: Presenting complaint among patients with myocardial infarction who present to an urban, public hospital emergency department. Ann Emerg Med 2002, 40:180-186.
14. Thompson DA, Eitel D, Fernandes CMB, Pines JM, Amsterdam J, Davidson SJ: Coded Chief Complaints-Automated Analysis of Free-text Complaints. Acad Emerg Med 2006, 13:774-782.

15. Ruger JP, Lewis $L M$, Richter $C$ J: Identifying high-risk patients for triage and resource allocation in the ED. Am J Emerg Med 2007, 25:794-798.

16. Safwenberg U, Terént A, Lind L: The Emergency Department presenting complaint as predictor of in-hospital fatality. Eur J Emerg Med 2007, $14: 324$.

17. Safwenberg U, Terént A, Lind L: Differences in Long-term Mortality for Different Emergency Department Presenting Complaints. Acad Emerg Med 2008, 15:9-16.

18. Schneider D, Appleton L, McLemore T: A reason for visit classification for ambulatory care. Vital Health Stat 2 1979, i-vi:1-63.

19. Niska R, Bhuiya F, Xu J: National hospital ambulatory medical care survey: 2007 emergency department summary. Stat Report: Natl Health; 2010:26.

20. Zakariassen E, Burman R, Hunskaar S: The epidemiology of medical emergency contacts outside hospitals in Norway - a prospective population based study. Scand J Trauma Resusc Emerg Med 2010, 18:9.

21. Huber CA, Rosemann T, Zoller M, Eichler K, Senn O: Out-of-hours demand in primary care: frequency, mode of contact and reasons for encounter in Switzerland. J Eval Clin Pract 2011, 17:174-179.

22. Chmiel C, Huber CA, Rosemann T, Zoller M, Eichler K, Sidler P, Senn O: Walk-ins seeking treatment at an emergency department or general practitioner out-of-hours service: a cross-sectional comparison. BMC Health Serv Res 2011, 11:94.

23. Christenson J, Innes G, McKnight D, Boychuk B, Grafstein E, Thompson CR, Rosenberg F, Anis AH, Gin K, Tilley J, et al: Safety and efficiency of emergency department assessment of chest discomfort. Can Med Assoc J 2004, 170:1803-1807.

24. Tong A, Sainsbury P, Craig J: Consolidated criteria for reporting qualitative research (COREQ): a 32-item checklist for interviews and focus groups. Int J Qual Health Care 2007, 19:349-357.

\section{doi:10.1186/1757-7241-20-76}

Cite this article as: Malmström et al:: Structured classification for ED presenting complaints - from free text field-based approach to ICPC-2 ED application. Scandinavian Journal of Trauma, Resuscitation and Emergency Medicine 2012 20:76.

\section{Submit your next manuscript to BioMed Central and take full advantage of:}

- Convenient online submission

- Thorough peer review

- No space constraints or color figure charges

- Immediate publication on acceptance

- Inclusion in PubMed, CAS, Scopus and Google Scholar

- Research which is freely available for redistribution 